\title{
Rabaska
}

Revue d'ethnologie de l'Amérique française

N'DA, PAUL. Alliances à plaisanterie, proverbes et contes en

Afrique de la tradition. Pour une société d'entraide et de justice. Paris, L'Harmattan, 2017, 133 p. ISBN 978-2-343-10653-3

\section{Patrice Kouraogo}

Volume 16, 2018

URI : https://id.erudit.org/iderudit/1051355ar

DOI : https://doi.org/10.7202/1051355ar

Aller au sommaire du numéro

Éditeur(s)

Société québécoise d'ethnologie

ISSN

1703-7433 (imprimé)

1916-7350 (numérique)

Découvrir la revue

Citer ce compte rendu

Kouraogo, P. (2018). Compte rendu de [N'DA, PAUL. Alliances à plaisanterie, proverbes et contes en Afrique de la tradition. Pour une société d'entraide et de justice. Paris, L’Harmattan, 2017, 133 p. ISBN 978-2-343-10653-3]. Rabaska, 16, 287-291. https://doi.org/10.7202/1051355ar d'utilisation que vous pouvez consulter en ligne.

https://apropos.erudit.org/fr/usagers/politique-dutilisation/ 
Franco-Amérique » (p. 50-54, paroles à l'appui), sur les communautés francoontariennes (p. 87-88) et sur Saint-Pierre-et-Miquelon (p. 250-251).

En ce qu'il puisse servir, ce livre nourrit le rêve commun d'une Francophonie multiple d'Amérique, métissée, stratifiée, éparpillée ; un rêve, non pas qu'il soit illusoire ou virtuel, mais parce qu'il est immanent aux quatre coins de ce continent.

YaÏVES FerLand

Université Laval

N'DA, Paul. Alliances à plaisanterie, proverbes et contes en Afrique de la tradition. Pour une société d'entraide et de justice. Paris, L'Harmattan, 2017, 133 p. ISBN 978-2-343-10653-3.

$\mathrm{Si}$, de façon classique, les chercheurs analysent très souvent séparément les alliances à plaisanterie, les proverbes et les contes comme des thématiques différentes, dans cette œuvre d'une belle facture, Paul N'Da a su faire un mélange savant de ces outils culturels et éducatifs pour faire passer plusieurs messages riches.

L'un de ces messages mis en exergue est l'extraordinaire capacité de régulation des tensions sociales de ces outils. Les alliances, les contes et les proverbes ont toujours servi de valeurs thérapeutiques dans la mesure où ils indiquent les voies de la réconciliation quelles que soient la nature et les raisons des conflits qui peuvent surgir dans une communauté humaine qui les exploite comme des moyens de socialisation, de formation et d'éducation.

Aussi, l'une des caractéristiques originales de l'œuvre réside dans son caractère transversal africain. En effet, nous avons lu une bonne partie des premières pages du livre avant de nous rendre compte qu'il s'agissait spécifiquement de l'ethnie Akan de la Côte d'Ivoire. En tant que Burkinabè, nous retrouvions une similitude parfaite avec l'ethnie moaaga du Burkina Faso, car les réalités décrites sont presque identiques. Naturellement, ces réalités sont valables dans plusieurs communautés socioculturelles africaines traditionnelles.

L'auteur a procédé selon une approche sociologique des éléments culturels phares d'une communauté de l'Afrique traditionnelle dont les fonctions socioculturelles peuvent être extrapolées. Cette déconstruction des fonctions et du rôle de ces outils pédagogiques nous semble pertinente en tant que spécialiste de la sociologie de la culture. Même si Paul N'Da table sur la sociologie politique, l'usage des matériaux culturels a son pesant d'or, car cette approche rejoint nos convictions que l'approche sociologique donne la possibilité non seulement de s'intéresser au passé, mais renseigne sérieusement sur l'utilité 
sociale actuelle des alliances à plaisanterie, des proverbes et des contes par le truchement de leur rôle en tant qu'outils de formation et d'éducation dans le présent et dans l'évolution des sociétés africaines.

L'œuvre de 133 pages du professeur titulaire des universités en sociologie politique est scindée en quatre chapitres dont l'examen approfondi permet de dégager ce qui suit.

Le premier chapitre, intitulé « Société d'entraide et de solidarité : régulation sociale et cohésion ", situe le lecteur sur la philosophie et le fondement conceptuel de la société d'entraide et de la solidarité dans le contexte africain. L'acquisition de l'assimilation et de la pratique de solidarité est une lutte perpétuelle, car elle est la base de la cohésion sociale. Parti de la conviction que la cohésion sociale est un combat de fond pour toute société, l'auteur soutient que les sociétés africaines de la tradition ont fondamentalement deux instances de régulation, qui sont les alliances à plaisanterie et les autres mécanismes de la thérapie contre les maux de cœur (rancunes, rancœur, le malheur, etc,).

La régulation par la thérapie de la réconciliation soigne la «maladie du cœur » que l'auteur prend le soin de traduire dans la langue maternelle de plusieurs ethnies africaines. La réconciliation serait le grand remède, surtout après la demande de pardon. Il faut donc guérir le «Sipè » (maladie du cœur), car il résiste au temps, ne se résorbe pas et ne disparaît pas de lui-même. La parenté et les alliances à plaisanterie sont des institutions existantes chez plusieurs peuples africains qui font que divers groupes et communautés deviennent des « frères » et « alliés », qu'ils renoncent à la possibilité de l'affrontement fratricide. La régulation par les alliances à plaisanterie évite que le « Sipè » ne saccage la cohésion et la réconciliation et cette régulation joue le rôle de purification des cœurs.

À côté du jeu de la parenté et des alliances à plaisanterie, d'autres formes de joutes verbales existent à travers les communautés du monde et Paul N'Da interroge leur fonction sociale réelle, sachant que le ridicule est craint, car il tue symboliquement, l'humiliation publique étant l'outrage ultime qui « verse le visage à terre » chez les Africains. Aussi, leur apport dans l'Afrique de la modernité aux prises avec des luttes pour la démocratie et la bonne gouvernance, et surtout pour des relations sociales et politiques apaisées, fait l'objet d'attention particulière.

La conviction est ferme que les États pourraient bien se plier à des accords, des traités, des alliances qui les condamneraient à refuser systématiquement le recours à la violence, au conflit ouvert, à la guerre comme modalité de règlement de conflit et de crise. C'est à ce prix que la politique pourra être un lieu de médiation centrale de la société et d'arbitrage des conflits sociétaux, au nom des valeurs de cohésion globale. Ainsi donc, des valeurs bien 
intégrées, parfaitement assimilées, autorisent le contrôle social sans qu'il soit nécessaire d'organiser toute une politisation des relations des acteurs de conflits (p. 29-30).

Le deuxième chapitre se penche sur les proverbes du groupe ethnique Akan et surtout cherche à appréhender les notions de l'ordre et du désordre. À ce niveau, c'est la perspective littéraire qui est privilégiée et permet de revisiter la pensée disjonctive de Descartes sur le noir et le blanc, sur le jour et la nuit pour se rendre à l'évidence que le gris, l'aurore et le crépuscule sont des réalités médianes qui évoquent la relativité des choses et la nécessité d'éviter les extrémités absolues qui conduisent volontairement à l'extrémisme tout court. On retient que le proverbe cherche à illustrer, à développer, à expliquer une contestation qui peut s'appliquer analogiquement à un fait et se construisait à partir de l'observation de faits naturels (d'existence ou de comportement). C'est alors que les proverbes plongent leurs racines dans la tradition qui observe, explique et interprète les faits, les règles, la nature des choses sociales, les comportements humains pour expliquer les relations sociales. Pour fluidifier ces relations, le concept de l'ordre devient central.

Partant du principe que la stabilité, la cohésion, la permanence et la reproduction sociale ne sont possibles que dans un monde ordonné, il faut enseigner la nécessité qu'il y a forcément un ordre dans le monde, un ordre des choses, des êtres, un ordre dans l'existence. De façon pragmatique donc, les parents et éducateurs doivent veiller à ce que « L'oiseau n'enfante la souris » et doivent expliquer que « c'est là où passe l'aiguille que passe le fil », que " si un enfant dit des injures, c'est qu'on les lui a apprises ", que " si la pensée ne le pense pas, la langue ne le dit pas » (p. 41). Aussi, avec une panoplie de proverbes instructifs bien choisis, Paul N'Da analyse la teneur éducative, contraignante et salvatrice de l'existence de l'ordre dans une société et surtout les retombées de son ensemencement par le truchement de son enseignement dans les jeunes esprits. En bref, pour qu'une société vive des rapports sociaux et interpersonnels loyaux, il faut qu'ils soient fondés sur la sincérité, le respect des statuts et des places. Le pouvoir est un moyen efficace d'assurer le contrôle social, de protéger et de maintenir l'ordre social au bénéfice de la communauté entière.

Les troisième et quatrième chapitres portent sur les contes, mais toujours et encore sur le concept de l'ordre qui semble être la pierre angulaire autant de la réconciliation que de la cohésion sociale. Un corpus de contes Akan sert d'appui à cette analyse.

Si un analyseur est un phénomène qui permet d'apercevoir et d'énoncer les contradictions d'une institution (Loureau, 1970 ; Hesse et Savoye, 1993), on doit admettre que le conte en est un exemple. La démarche consiste à 
utiliser les contes comme des discours de légitimation de l'ordre. Le conte est un moyen de socialisation très apprécié, car il transmet pratiquement l'ensemble des valeurs culturelles, des normes de comportement. Il est un analyseur sociologique dans la mesure où il part et parle de la réalité sociale, et atteint la réalité sociologique par une recherche de réseaux applicatifs de relations. Le conte révèle nettement l'existence, d'un côté, de groupes dominants, ceux qui ont le pouvoir de mobiliser les institutions et les valeurs pour défendre leurs intérêts et assurer la reproduction de leur privilège, et, de l'autre, de groupes dominés pour lesquels l'essentiel est de se défendre, de visiter et de protester. Dans le conte, les dominés sont représentés par le pauvre, le faible, l'orphelin, la femme, l'enfant, le lièvre, l'araignée, etc. Les dominants sont les notables, le roi, les riches, les chanceux, les génies, Dieu, l'éléphant, le lion, la panthère, jusqu' aux parents et la communauté villageoise qui participent en position avantageuse à l'ordre établi (p. 56). Par des contes bien choisis, l'auteur prône la promotion de l'ordre qui seul peut garantir la cohésion sociale. Ainsi, les thèmes de paix, de refus de l'anarchie, de pouvoir sont abordés dans des contes pris pour exemples. Il faut donc que chaque individu de la société apprenne les valeurs de l'ordre social à respecter, perçu comme un chemin tracé déjà par les ancêtres et les parents.

Pendant que la soumission et la docilité sont présentées comme valeurs positives parce qu'elles forment aux normes imminentes de la communauté, la désobéissance pactise avec le désordre, puisque celui qui refuse d'obéir à une injonction s'attaque à l'ordre social. La morale des contes est à souhait conformiste (p. 7). La société traditionnelle africaine est fortement consciente de son unité, centrée sur les valeurs et la recherche du consensus. Elle paraît phallocratique et gérontocratique : les hommes et les vieux y sont plus valorisés que les femmes et les vieilles. Les normes sociales des groupes dominants et dirigeants donnent les orientations culturelles et sont des instruments de reproduction de l'ordre établi. Le conte est essentiellement le combat des dominés et il prête un pouvoir particulier aux malades, aux faibles, aux êtres en marge de la société.

Le quatrième chapitre cible les notions de domination, de ruse et de refus. Ce chapitre montre comment la société, à travers ses contes, utilise la ruse et la dérision comme armes pour mener les luttes sociales et politiques pour une société décente. Sur le constat que le conte traduit les mouvements d'ordre et de désordre au sein de la société et que ses instruments préférés sont la ruse et la dérision, la dénonciation sociale et politique y est exprimée à travers la critique des pratiques et en particulier d'attitudes à l'égard des petits, des sans-pouvoir. Partant du principe que la dérision porte au rire, démystifie les puissants, les ridiculise en effaçant leur grandeur, leur pompe, et que la ruse 
est faite d'intelligence, de sagesse, de débrouillardise, d'habileté, de malice, de duplicité, de sensibilité intuitive, le conte éduque, forme et transforme l'individu et la collectivité. Il appelle et apporte des rectifications, des amendements, des modifications nécessaires.

Les luttes sociales et politiques n'ont un sens que d'en appeler à une " société de semblables », comme une « société des égaux » (Rosanvollon, p. 119). La société décente, bâtie sur le socle de la dignité sociale, d'une éthique et d'une politique de la reconnaissance et d'une « clinique de l'injustice » est l'idéal à réaliser, et le conte devient, dans ce sens, un instrument incontournable.

Patrice Kouraogo

Institut des sciences de sociétés (INSS/CNRST), Ouagadougou

Paradis, Clara. Souvenirs de ma dernière année au pensionnat St-Roch, Québec, 1894-1895. Journal personnel d'étudiante tenu dans un pensionnat de jeunes filles de la Basse-ville de Québec à la fin du XIXe siècle. Texte présenté, annoté et édité par Gilles PAGEAU, Québec, $2^{\mathrm{e}}$ tirage 2009, 372 p. ISBN non inscrit.

À quoi rêvaient les jeunes filles qui séjournaient dans un pensionnat à la fin du XIX ${ }^{e}$ siècle ? Le journal personnel de Clara Paradis nous en donne un aperçu malheureusement limité. Car on réalise rapidement à la lecture de ce journal rédigé dans le cadre d'un pensionnat tenu par des religieuses qu'il valait mieux pour les pensionnaires de ne pas trop s'épancher sur leurs états d'âme, les religieuses exerçant fort probablement une surveillance étroite sur les écrits de leurs pupilles. Ce journal s'avère tout de même fort intéressant, car il nous donne accès au quotidien et aux préoccupations d'une jeune fille de 19 ans qui s'apprête à quitter le pensionnat Saint-Roch, situé dans la BasseVille de Québec, après y avoir séjourné pendant quatre ans. Dans ses écrits journaliers, s'échelonnant de septembre 1894 à juin 1895, Clara Paradis relate les principaux événements survenus au pensionnat et nous donne un aperçu de l'atmosphère qui régnait dans ces institutions réservées aux jeunes filles. Il s'en dégage une vision plutôt positive de la vie au pensionnat. Manifestement, Clara Paradis y a vécu des jours heureux, entourée de ses compagnes et des religieuses de la Congrégation Notre-Dame qui avaient la charge de cet établissement. Le journal personnel de Clara Paradis nous livre de nombreuses informations concernant la vie au pensionnat : les matières enseignées, les lectures imposées, les récompenses et punitions, les sorties, les loisirs, les visites au parloir et les nombreuses pratiques religieuses qui ponctuaient le quotidien des pensionnaires. Quelques passages particulièrement émouvants 